\title{
The Role of Acceptance and Commitment-based Group Therapy on Pain Tolerance and State-trait Anxiety in Gastrointestinal Cancer Patients
}

\author{
Mahdi Fatemizadeh ${ }^{1}$, Farideh Ghavasi ${ }^{2}$, Benyamin Siran Hesari ${ }^{3}$, Iman Akbarzadeh ${ }^{4}$, \\ Nasim Nadi Moghadam ${ }^{1}$ and Masoomeh Shirzad ${ }^{5, *}$
}

${ }^{1}$ Department of Neuroscience and Addiction Studies, School of Advanced Technologies in Medicine, Tehran University of Medical Sciences, Tehran, Iran

${ }^{2}$ Department of Psychology, Islamic Azad University of Bojnourd, North Khorasan, Iran

${ }^{3}$ Department of English language, Faculty of Teaching English as a Foreign Language, Imam Reza International University, Mashhad, Iran

${ }^{4}$ Department of Chemical and Petroleum Engineering, Biotechnology Research Center, Sharif University of Technology, Tehran, Iran

${ }^{5}$ Drug Design and Bioinformatics Unit, Medical Biotechnology Department, Biotechnology Research Center, Pasteur Institute of Iran, Tehran, Iran

\begin{abstract}
The main purpose of this study was to investigate the impact of acceptance and commitment group therapy on pain tolerance and state-trait anxiety in patients with gastrointestinal cancer. This quasi-experimental study was performed with pretest, posttest and control group. There were 24 participants in the study, 12 of them in the control group and other 12 in the experimental group. The experimental group received eight sessions of acceptance and commitment based therapy and the control group received no psychotherapy. The instruments used in this study were short-form McGill Pain questionnaire-2 and state-trait anxiety questionnaire. Results showed an increase in pain tolerance and decrease in anxiety in patients in the experimental group.
\end{abstract}

Keywords: Group therapy, acceptance and commitment therapy, gastrointestinal cancer, pain tolerance, anxiety.

\section{INTRODUCTION}

Despite remarkable advances in the medical disciplines, cancer still remains as one of the most significant diseases of present time and its mortality rate is high. There are also more than 200 types of identified cancers at the moment, with an increasing incidence, while it is estimated that more than 15 million people in the world will be infected by 2020 [1].

Cancer has many effects on the community [2] in a way that the infected people might suffer from anxiety, depression, cognitive impairment and other physical and psychological symptoms [3] all of which affect one's function in the society. Moreover, the psychological and physical symptoms of this disorder decrease one's independence and ability to balance and influence the family environment, leading to uncertainty and lack of self-esteem [4]. Besides, uncertainty anxiety and poor prognosis of cancer can cause a person, during their illness, to suffer from higher anxiety than ordinary people in the society,

*Address correspondence to this author at the Drug Design and Bioinformatics Unit, Medical Biotechnology Department, Biotechnology Research Center, Pasteur Institute of Iran, Tehran, Iran, E-mail: masishirzad@hotmail.com whose high anxiety side effects have devastating impacts on psychological and physical symptoms, leading to negative effect of one's performance in society [5].

Cancer itself causes psychological problems for most patients, and these psychological symptoms will have a detrimental effect on their other functions. Removing these problems in cancer patients can make them more resistant to the unpleasant symptoms of cancer.

Due to problems with this type of cancer and current medical treatments, these patients suffer from high levels of pain and this level of pain also causes psychological problems such as high levels of dissatisfaction, anxiety, and depression.

Currently, gastrointestinal cancer is one of the most common causes of death among other cancers, and it creates wide-ranging psychological symptoms in the patient [6]. Numerous studies have also indicated high levels of anxiety in gastrointestinal cancer patients [7].

Acceptance and commitment approach therapies accentuate the issue of communication system in the 
psychological problems of human beings and are considered to be very effective in the issues that lead to resentment or irritation. This therapeutic approach also considers the avoidance of pain and stress as a major problem for humans, which can lead to disability and reduced life satisfaction. It is asserted by this approach that avoidance occurs when negative thoughts and emotions exert an excessive and inappropriate effect on behavior. Therefore, the therapeutic approach efforts purpose, based on acceptance and commitment, is to expose the patients to conditions and situations that they mostly avoid [8].

Acceptance and commitment-based treatment focuses more on the acceptance of negative symptoms from avoidant situations such as illnesses rather than controlling the symptoms [8, 9]. The goal of psychotherapy based on acceptance and commitment is to improve individuals' performance by increasing their psychological flexibility [10]. Studies by other researchers demonstrate the fact that this approach has a positive effect on enhancing physical and emotional performance [11].

\section{METHODOLOGY}

In this quasi-experimental study, 24 men with gastro-intestinal cancer in Tehran were used. All samples were selected by convenience sampling. The inclusion criteria for the study subjects were ages between 20 to 65 , minimum reading and writing age, and no separate psychotherapy involvement during the study. Exclusion criteria were also altering therapeutic medicine during group therapy and receiving separate psychotherapy.

Initially, all participants were randomly divided into two groups of experimental and control. And after the registration, the first session was held separately for each participant to acquaint with the study process. Pain and state-trait anxiety questionnaires were administered to all participants. The experimental group underwent acceptance and commitment based therapy weekly and the control group did not receive group psychotherapy intervention until the end of the study.

Acceptance and commitment therapy was implemented based on Hayes protocol [12]. This psychotherapy protocol relies on eight sessions that in each session, a specific content is provided. In the initial sessions, people in the group become more familiar with each other and the therapeutic relationship between the group and the members is formed. It discusses issues such as confidentiality and therapeutic experiences during cancer. Feedback from other members is also an important point of the group. Concepts such as the dysfunctional system and "controlling the problem is not the solution" are discussed. The metaphors and fundamental concepts of the Acceptance and Commitment Approach are also discussed in the following sessions, some tasks are given to participants throughout the week as their homework, and they are taught skills such as mindfulness or meditation. The closing sessions also discuss the story of personal life by emphasizing the present time and commitment in conducting the activities and lessons which were taught. Each person narrates his own experiences and receives feedback from the members of the group.

At the end the questionnaires are collected from every participant.

\section{Short form McGill Pain Questionnaire-2 (SF-MPQ-2)}

This questionnaire was designed to assess the quality of pain severity and it is capable of measuring the neuropathic pain and non-neuropathic pain [13]. The questionnaire consists of 22 questions ranging from 0 to 10 . Zero means no pain and 10 means the worst possible pain in the week before. This questionnaire contains four subsets including Continuous pain, Intermittent pain, Neuropathic pain and Affective pain [14].

\section{Spielberger State-Trait Anxiety Inventory (STAI-Y)}

The Spielberg Anxiety Inventory consists of 40 questions that the first 20 items measure the state of anxiety and the second 20 items measure the trait of anxiety. The State of Anxiety Scale (explicit anxiety) is consisted of 20 sentences that examine one's emotions at the moment and the time of response. Anxiety trait scale (hidden anxiety) also includes 20 sentences that measure one's general and typical emotions. The response scale is also ranged from 1 very low to 4 very high [15].

\section{RESULT}

The purpose of this study was to evaluate the effectiveness of group psychotherapy based on acceptance and commitment on pain and anxiety tolerance in patients with gastrointestinal cancer. Participants were 12 men with gastrointestinal cancer with a mean of 51 years (SD: 5.69). Table 1 also shows 
Table 1: Frequency of Participants Qualifications

\begin{tabular}{|c|c|c|c|c|}
\hline & Frequency & Percent & Valid Percent & Cumulative Percent \\
\hline \hline Below Diploma & 2 & 8.3 & 8.3 & 8.3 \\
\hline Diploma & 10 & 41.7 & 41.7 & 50.0 \\
\hline Bachelor Degree & 9 & 37.5 & 37.5 & 87.5 \\
\hline Above Bachelor & 3 & 12.5 & 12.5 & 100.0 \\
\hline Total & 24 & 100.0 & 100.0 & \\
\hline
\end{tabular}

Table 2: Mean and Standard Deviation scores of pain tolerance and anxiety in Experiment and Control Groups

\begin{tabular}{|c|c|c|c|c|}
\hline \multirow{2}{*}{ Variable } & \multicolumn{2}{|c|}{ Experimental group } & \multicolumn{2}{c|}{ Control group } \\
\cline { 2 - 5 } & Pre-test & Post-test & Pre-test & Post-test \\
\hline \hline Pain & $162.5 \pm 8.9$ & $134.0 \pm 6.68$ & $175.25 \pm 1209$ & $166.0 \pm 8.65$ \\
\hline Anxiety & $106.08 \pm 6.35$ & $82.58 \pm 5.96$ & $99.08 \pm 7.02$ & $97.58 \pm 6.35$ \\
\hline
\end{tabular}

the frequency of their education. Among all the participants, the frequency of diploma with $41 \%$ was the highest.

Covariance analysis was used to analyze the data. But first, Kolmogorov-Smirnov test was used to check the normality of the factors. The result of KolmogorovSmirnov test was not significant, indicating that the distribution of test variables was normal. Therefore, the assumptions of normality of the factors were accepted. Levene test was also used to confirm the homogeneity of variances hypothesis. The obtained results showed that the variances were equal.
Table 4 shows that anxiety and pain intensity were significantly decreased in the experimental group after the intervention compared to the control group. The findings of this study showed that gastrointestinal cancer patients demonstrated lower levels of anxiety and pain intensity after being admitted to the treatment group based on acceptance and commitment.

\section{DISCUSSION}

Cancer disease is one of the critical issues of all societies that besides the physical problems it causes many psychological injuries such as tension and

Table 3: Levene Test for Homogeneity of Variances

\begin{tabular}{|c|c|c|c|}
\hline variables & Degree of freedom1 & Degree of freedom2 & Significant \\
\hline \hline Pre-anxiety & 1 & 22 & 0.93 \\
\hline Post-anxiety & 1 & 22 & 0.63 \\
\hline Pre-pain & 1 & 22 & 0.48 \\
\hline Post-pain & 1 & 22 & 0.54 \\
\hline
\end{tabular}

Table 4: Analysis of Covariance to Compare Two Groups Pre-Test and Post-Test

\begin{tabular}{|c|c|c|c|c|c|c|}
\hline Indicators & Source & Degree of freedom & Mean Square & F & P Value & Partial Eta Square \\
\hline \hline \multirow{3}{*}{ Anxiety } & Pre-test & 1 & 294.00 & 6.548 & 0.018 & 0.229 \\
\cline { 2 - 7 } & Post-test & 1 & 1350.00 & 35.53 & 0.000 & 0.618 \\
\cline { 2 - 8 } & error & 22 & 37.992 & & 0.008 & 0.282 \\
\hline \multirow{3}{*}{ pain } & Pre-test & 1 & 975.375 & 8.620 & 0.000 & 0.824 \\
\cline { 2 - 8 } & Post-test & 1 & 6144.00 & 102.711 & & \\
\cline { 2 - 7 } & error & 22 & 59.818 & & & \\
\hline
\end{tabular}


anxiety for the patient [16]. These patients, in addition to having physical symptoms, face anxiety that may often be plagued with recurrent unpleasant thoughts, such as fear of pain, death, and dependence on others. The type of an anxious patient thinking is characterized by extroversion and catastrophizing. Negative results seem to be inevitable, and patients find themselves in a hopeless condition [17].

Therefore, the psychological support of these patients is of great importance. Psychological support generally has two aspects of behavioral therapy and drug therapy. In drug therapy with sedation such as benzodiazepines, the patient faces with many side effects, including dry mouth, dizziness, constipation, and the risk of overdose and dependence. Even lowerrisk drugs, such as buspirone, have side effects such as dizziness, drowsiness, and nausea while no adverse effects have been reported in behavioral treatment [18].

In the present study, the effects of acceptance and commitment based group therapy on pain tolerance and state-trait anxiety in gastrointestinal patients were investigated. The results indicated that the mentioned method had positive effects on increasing tolerance and reducing pain and anxiety in the experimental group after the intervention compared to the control group. The results of this study are in line with studies by Staci Martin et al. [19]. on neurofibromatosis type 1 patients, mohabbat-bahar in improving anxiety symptoms in patients with breast cancer [20] and with Arch et al. It has been shown to reduce anxiety and depression and improve the pain component in cancer survivors, which have been identified as the most effective strategies for controlling anxiety in people with cancer, psychotherapy, drug therapy and behavioral therapy [20].

Roth and Massie [21] and Hulbert [22] in their paper, similar results were reported and they acknowledged that patients who received pain tolerance treatment and were aware of the nature of their pain experienced less anxiety and stress. In this study, acceptance and commitment-based group therapy methods were used that gives patients the ability to relate to their experience here and now [23].

The reason could be due to the fact that this therapeutic approach strengthens the commitment and acceptance of cognitive processes and helps the patient to accept and deal with their moment-tomoment problems and emotions and to be more hopeful about the future [24].

\section{CONCLUSION}

Based on the results of the present study and the previous studies, effects of group therapy on pain and positive anxiety have been reported. Therefore, acceptance-based therapy and commitment can be considered as one of the best ways to control the anxiety and pain caused by gastrointestinal cancer.

Acceptance and commitment-based therapeutic approach stemming from its treatment philosophy, puts more emphasis on the components of here and now and acceptance of the present conditions. Given the annoying symptoms of gastrointestinal cancer, this approach can be considered as one of the best therapeutic approaches compared to drug therapy and other psychotherapeutic approaches.

\section{REFERENCES}

[1] Higginson IJ, Costantini M. Dying with cancer, living well with advanced cancer. European journal of cancer (Oxford, England : 1990) $2008 \mathrm{Jul} ;$ 44(10): 1414-24. https://doi.org/10.1016/j.ejca.2008.02.024

[2] Costa DS, Mercieca-Bebber R, Rutherford C, Gabb L, King MT. The impact of cancer on psychological and social outcomes. Australian Psychologist 2016; 51(2): 89-99. https://doi.org/10.1111/ap.12165

[3] Jensen W, Baumann FT, Stein A, Bloch W, Bokemeyer C, de Wit $M$, et al. Exercise training in patients with advanced gastrointestinal cancer undergoing palliative chemotherapy: a pilot study. Supportive Care in Cancer 2014; 22(7): 1797-806. https://doi.org/10.1007/s00520-014-2139-x

[4] Chambers SK, Baade P, Youl P, Aitken J, Occhipinti S Vinod $S$, et al. Psychological distress and quality of life in lung cancer: the role of health-related stigma, illness appraisals and social constraints. Psycho-Oncology 2015; 24(11): 1569-77. https://doi.org/10.1002/pon.3829

[5] Canxia X, Shen S, Ling Q. A Discussion about Effect of Psychological Factor and Social Factor on the Prevention and Treatment of Gastrointestinal Tumor. Chinese Journal of Clinical Psychology 1993 (02).

[6] Winterling J, Wasteson E, Arving C, Johansson B, Glimelius B, Nordin K. Factors associated with psychological distress and grief resolution in surviving spouses of patients with advanced gastrointestinal cancer. Supportive care in cancer 2010; 18(11): 1377-84.

https://doi.org/10.1007/s00520-009-0753-9

[7] Chung J, Ju G, Yang J, Jeong J, Jeong Y, Choi MK, et al. Prevalence of and factors associated with anxiety and depression in Korean patients with newly diagnosed advanced gastrointestinal cancer. The Korean journal of internal medicine 2018; 33(3): 585. https://doi.org/10.3904/kjim.2016.108

[8] Hiratzka J, Rastegar F, Contag AG, Norvell DC, Anderson PA, Hart RA. Adverse event recording and reporting in clinical trials comparing lumbar disk replacement with lumbar fusion: a systematic review. Global spine journal 2015; 5(06): 486-95. https://doi.org/10.1055/s-0035-1567835

[9] Hayes SC, Strosahl KD. A practical guide to acceptance and commitment therapy: Springer Science \& Business Media; 2004.

https://doi.org/10.1007/978-0-387-23369-7 
[10] Prevedini AB, Presti G, Rabitti E, Miselli G, Moderato P. Acceptance and commitment therapy (ACT): the foundation of the therapeutic model and an overview of its contribution to the treatment of patients with chronic physical diseases. G Ital Med Lav Ergon 2011; 33(1 Suppl A): A53-A63.

[11] Vowles KE, McCracken LM, O'Brien JZ. Acceptance and values-based action in chronic pain: a three-year follow-up analysis of treatment effectiveness and process. Behaviour research and therapy $2011 ; 49(11): 748-55$. https://doi.org/10.1016/j.brat.2011.08.002

[12] Hayes SC, Levin ME, Plumb-Vilardaga J, Villatte JL, Pistorello J. Acceptance and commitment therapy and contextual behavioral science: Examining the progress of a distinctive model of behavioral and cognitive therapy. Behavior therapy 2013; 44(2): 180-98. https://doi.org/10.1016/j.beth.2009.08.002

[13] Dworkin RH, Turk DC, Revicki DA, Harding G, Coyne KS, Peirce-Sandner $\mathrm{S}$, et al. Development and initial validation of an expanded and revised version of the Short-form McGill Pain Questionnaire (SF-MPQ-2). PAIN® 2009; 144(1-2): 3542. https://doi.org/10.1016/j.pain.2009.02.007

[14] Kachooei AR, Ebrahimzadeh MH, Erfani-Sayyar R, Salehi M, Salimi E, Razi S. Short Form-McGill Pain Questionnaire-2 (SF-MPQ-2): a cross-cultural adaptation and validation study of the Persian version in patients with knee osteoarthritis. Archives of bone and joint surgery 2015; 3(1): 45.

[15] Spielberger CD. State-Trait anxiety inventory. The Corsini encyclopedia of psychology 2010: 1. https://doi.org/10.1002/9780470479216.corpsy0943

[16] Zeynalova N, Schimpf S, Setter C, Yahiaoui-Doktor M, Zeynalova S, Lordick F, et al. The association between an anxiety disorder and cancer in medical history. Journal of affective disorders 2019; 246: 640-2. https://doi.org/10.1016/j.jad.2018.12.019

[17] Mirzaeidoostan Z, Zargar Y, Zandi Payam A. The Effectiveness of Acceptance and Commitment Therapy on Death Anxiety and Mental Health in Women With HIV in
Abadan City, Iran. Iranian Journal of Psychiatry and Clinical Psychology 2019; 25(1): 2-13.

https://doi.org/10.32598/ijpcp.25.1.2

[18] Gale C, Oakley-Browne M. Anxiety disorder. BMJ 2000; 321(7270): 1204-7.

https://doi.org/10.1136/bmj.321.7270.1204

[19] Martin S, Wolters PL, Toledo-Tamula MA, Schmitt SN Baldwin A, Starosta A, et al. Acceptance and commitment therapy in youth with neurofibromatosis type 1 (NF1) and chronic pain and their parents: A pilot study of feasibility and preliminary efficacy. American journal of medical genetics Part A 2016; 170(6): 1462-70. https://doi.org/10.1002/ajmg.a.37623

[20] Mohabbat-Bahar S, Maleki-Rizi F, Akbari ME, Moradi-Joo M. Effectiveness of group training based on acceptance and commitment therapy on anxiety and depression of women with breast cancer. Iranian journal of cancer prevention 2015; 8(2): 71.

[21] Roth AJ, Massie MJ. Anxiety and its management in advanced cancer. Current Opinion in supportive and palliative care $2007 ; 1(1): 50-6$. https://doi.org/10.1097/SPC.0b013e32813aeb23

[22] Hulbert-Williams NJ, Storey L, Wilson KG. Psychological interventions for patients with cancer: psychological flexibility and the potential utility of Acceptance and Commitment Therapy. European journal of cancer care 2015; 24(1): 1527. https://doi.org/10.1111/ecc.12223

[23] Forsyth JP, Eifert GH. The mindfulness and acceptance workbook for anxiety: A guide to breaking free from anxiety, phobias, and worry using acceptance and commitment therapy: New Harbinger Publications; 2016.

[24] Mahmoudi F, Maddahi M, Poursharifi H, Meschi F. Comparison of the Effectiveness of Acceptance and Commitment Group Therapy and Cognitive-Behavioral Group Therapy on Quality of Life, Anxiety and Depression in Patients with Functional Indigestion. Journal of Health Promotion Management 2019; 8(4): 43-52.

Received on 18-11-2019 Accepted on 26-12-2019

Published on 30-12-2019

https://doi.org/10.30683/1927-7229.2019.08.08

(C) 2019 Heidari et al:; Licensee Neoplasia Research.

This is an open access article licensed under the terms of the Creative Commons Attribution Non-Commercial License (http://creativecommons.org/licenses/by-nc/3.0/) which permits unrestricted, non-commercial use, distribution and reproduction in any medium, provided the work is properly cited. 